\begin{tabular}{|c|c|}
\hline Title & Homogeneous nano-patterning using plasmon- assisted photolithography \\
\hline Author(s) & Ueno, Kosei; Takabatake, Satoaki; Onishi, Ko; Itoh, Hiroko; Nishijima, Y oshiaki; Misawa, Hiroaki \\
\hline Citation & $\begin{array}{l}\text { A pplied Phy sics Letters, 99(1), } 011107 \\
\text { https://doi.org/10.1063/1.3606505 }\end{array}$ \\
\hline Issue Date & 2011-07-04 \\
\hline Doc URL & http:/hdl.handle.net/2115/46881 \\
\hline Rights & $\begin{array}{l}\text { Copyright } 2011 \text { A merican Institute of Physics. This article may be downloaded for personal use only. A ny other use } \\
\text { requires prior permission of the author and the A merican Institute of Physics. The following article appeared in A ppl. } \\
\text { Phys. Lett. } 99,011107 \text { (2011) and may be found at https://dx.doi.org/10.1063/1.3606505 }\end{array}$ \\
\hline Type & article \\
\hline File Information & A PL99-1_011107.pdf \\
\hline
\end{tabular}

Instructions for use 


\title{
Homogeneous nano-patterning using plasmon-assisted photolithography
}

\author{
Kosei Ueno, ${ }^{1,2, a)}$ Satoaki Takabatake, ${ }^{1}$ Ko Onishi, ${ }^{1}$ Hiroko Itoh, ${ }^{1}$ Yoshiaki Nishijima, ${ }^{1}$ \\ and Hiroaki Misawa ${ }^{2, a)}$ \\ ${ }^{1}$ Research Institute for Electronic Science, Hokkaido University, Sapporo 001-0021, Japan \\ ${ }^{2}$ PRESTO, Japan Science and Technology Agency, Kawaguchi 332-0012, Japan
}

(Received 11 February 2011; accepted 12 April 2011; published online 6 July 2011)

\begin{abstract}
We report an innovative lithography system appropriate for fabricating sharp-edged nanodot patterns with nanoscale accuracy using plasmon-assisted photolithography. The key technology is two-photon photochemical reactions of a photoresist induced by plasmonic near-field light and the scattering component of the light in a photoresist film. The scattering component of the light is a radiation mode from higher order localized surface plasmon resonances scattered by metallic nanostructures. (C) 2011 American Institute of Physics. [doi:10.1063/1.3606505]
\end{abstract}

Near-field lithography holds promise for the fabrication of nano-patterns on a photoresist. ${ }^{1-6}$ Recently, we reported on the nano-patterning of pits ( $5 \mathrm{~nm}$ in diameter) onto a positive photoresist locally exposed by nanogap-assisted surface plasmon nanolithography. ${ }^{6}$ For near-field lithography, however, nano-patterns on a photoresist film are completely defined by the near-field intensity profile. As a result, photoresist patterns do not reflect the photomask design exactly. The near-field intensity profile can be predicted using computational electrodynamics modeling, such as finite-difference time-domain (FDTD) methods. ${ }^{7}$ However, the fabricated nano-patterns are shallow and highly dependent on exposure dose.

In this letter, we demonstrate a plasmon-assisted photolithography system that is used to fabricate nano-patterns with nanoscale accuracy. The most attractive feature of this system is its ability to form deep nano-patterns on positive photoresist. These formed nano-patterns accurately reflect the photomask's design. Our strategy for making deep nanopatterns involves the use of the radiation mode of localized surface plasmon resonance (LSPR) scattered on nanostructures onto photomasks as the exposure step. To make nanopatterns that accurately preserved our photomask design, we utilized a higher order LSPR mode with multi-wave functions. ${ }^{8}$ This mode was used to produce homogeneous scattering light in an $\mathrm{x}-\mathrm{y}$ plane that propagated the photoresist film along the z-axis. The key to making fine nano-patterns is to utilize scattered light based on a higher order of LSPR and two-photon photochemical reactions in the photoresist. This procedure promotes spatially selective exposure. Most important, we designed the photomasks using FDTD simulations. In addition, the lift-off process after the deposition of the metals onto the fabricated nano-patterns makes it possible to duplicate metallic nanostructures on a substrate. This procedure can be applied to plasmonic materials.

Gold-film-coated nanostructured photomasks were prepared by electron-beam (EB) lithography via sputtering onto glass substrates and by being brought into direct contact with a positive photoresist film spin-coated onto a glass substrate $\left(70 \mathrm{~nm}\right.$ in thickness). ${ }^{9}$ For the photomask fabrication process,

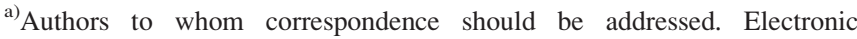
addresses: k-ueno@es.hokudai.ac.jp and misawa@es.hokudai.ac.jp.
}

nanostructures were directly formed using a negative EB resist on the substrate. ${ }^{9}$ Then, a 10-nm-thick gold film was deposited onto a nanostructured substrate. A scanning electron microscope (SEM) image of the photomask is shown in Figure 1(a). Rectangular cuboids were aligned along the substrate with a periodicity of $300 \mathrm{~nm}$. The extinction spectrum of the nanoblocks on the photomask and the absorption spectrum of the positive photoresist are indicated in Figure 1(b) (black and red lines, respectively). The plasmon resonance band in Figure 1(b) extends to near-infrared wavelengths, and there exists a small shoulder peak around $780 \mathrm{~nm}$. When a femtosecond laser beam with a center wavelength of $800 \mathrm{~nm}$ is used as an exposure source to irradiate the photoresist film, ${ }^{9}$ the photoresist is not excited directly, and only the shoulder peak of the plasmon band can be excited because of induced quadrupole resonance, as shown in the FDTD simulation results of Figure 1(c). ${ }^{9}$ The FDTD results in Figure 1(c) show the directions of the plasma electrons. The electric field along the $\mathrm{x}$-axis $\left(\mathrm{E}_{\mathrm{x}}\right)$ is represented by colors that correspond to the inset color bar at 0 and 1.33 fs after LSPR excitation. These plasma oscillations, induced by LSPR, continue up to approximately $20 \mathrm{fs}$. Thus, the relatively long dephasing time of the LSPR and localization of the electromagnetic (EM) field induce near-field enhancement effects $\left(|\mathrm{E}|^{2} \sim 100\right)$ at the corner of the gold nanoblocks along the polarization direction (x-axis). These effects are demonstrated by the near-field intensity profile obtained from the FDTD simulations in Figure 1(d) (highlighted by a dashed red circle). It should be noted that the propagating light in the photoresist film can be observed $0.67 \mathrm{fs}$ after plasmon excitation $(1 / 2 \pi$ phase shifted), as shown in Figure 1(d) (highlighted with dashed yellow lines). The observed light is due to the radiation mode from the higher order LSPRs scattered by the gold nanostructures. The EM enhancement factors $\left(|\mathrm{E}|^{2}\right)$ obtained from the FDTD simulations demonstrate an enhancement factor of twenty-one at the metal surface, six at the middle of the photoresist layer and two at the bottom of the photoresist layer. It was concluded that the intense scattering from the gold nanostructures on the photomask induces two-photon photochemical reactions within the photoresist.

Figures 1(e) and 1(f) show top and cross-sectional SEM images of the developed photoresist substrate. Homogeneous nano-patterns and the patterns reflected onto the photomask's 

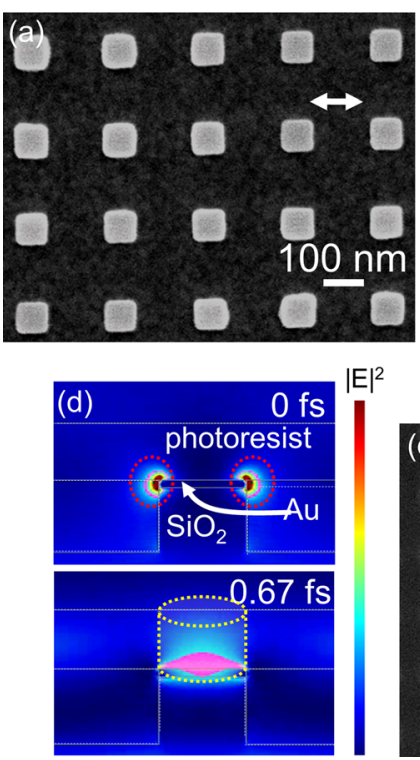

$|E|^{2}$

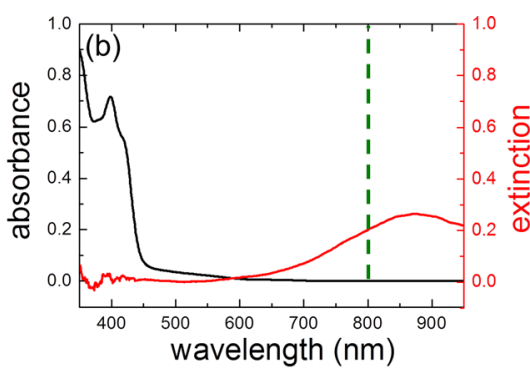

(c)
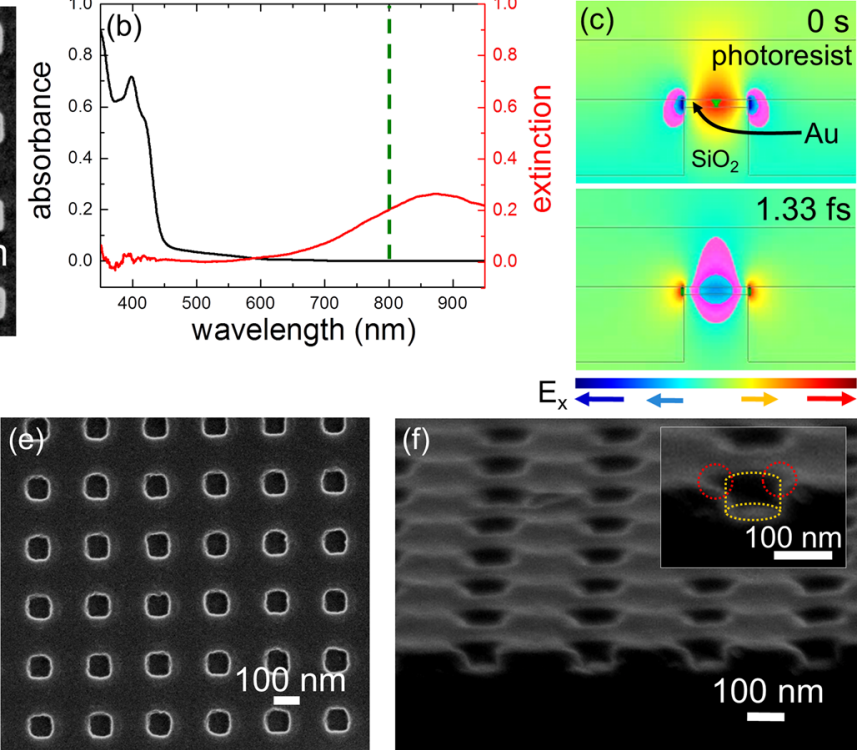

FIG. 1. (Color) (a) SEM image of a nanostructured photomask fabricated. Each block size is $100 \mathrm{~nm} \times 100 \mathrm{~nm} \times 100 \mathrm{~nm}$. The inset arrow shows the incident polarization direction during exposure. (b) Extinction spectrum of nanoblocks on the photomask (red line) and absorption spectrum of a positive photoresist (black line). (c) Schematic illustrations of the direction of plasma electrons and the electric field (Ex). (d) Electric field intensity profiles of the exposure setup at $0 \mathrm{fs}$ and $0.67 \mathrm{fs}$ after LSP excitation. (e) SEM image of the developed substrate (top view) after being exposed to a $50-\mathrm{W} / \mathrm{cm}^{2}$ laser beam for $10 \mathrm{~s}$ and after being developed in a developer solution (NMD-3, Tokyo Ohka Kogyo Co.) for 10 s. (f) A cross-sectional SEM image of the developed substrate. The inset figure shows an enlarged view of a pattern that includes exposure profiles with two components, as indicated by two dashed lines in different colors.

design directly were observed. It is worth mentioning that the cross-sectional SEM image has exposure profiles with two components, as indicated by the two dashed lines in Figure 1(f). Note that the exposure area at the resist surface, highlighted using red lines in Figure 1(f), corresponds to the near-field light component. In addition, the yellow lines in Figure 1(f) correspond to the scattering component of the light in the photoresist film. Through exposure and using the scattering component of the light, nano-patterns with a variety of sizes and shapes (i.e., chains, lines, and triangles) can be fabricated. Typical SEM images of the nano-patterns and their photomasks are provided in Figure 2. Chains containing six $150 \times 150 \mathrm{~nm}^{2}$ rectangular blocks include five bottlenecks with 10-nm-wide chains, as shown in Figure 2(b). These bottlenecks were confirmed to be all in an exposure area of $30 \times 30 \mu \mathrm{m}^{2}$ (1445 bottlenecks). Note that the standard deviation for the side length of the triangle patterns in Figure 2(d) was estimated to be $4.4 \mathrm{~nm}$ (average size is $141.2 \mathrm{~nm}$ ), and for the photomask (Figure 2(c)), it was 5.5 $\mathrm{nm}$ (average size is $145.1 \mathrm{~nm}$ ). Simultaneously, we estimated the curvature radius for the triangles at $90^{\circ}$ positions. The mean value measured was $16.6 \mathrm{~nm}$, and the standard deviation was estimated to be $2.8 \mathrm{~nm}$. These results indicate that highly accurate patterns can be fabricated using the lithography system. Most important, such nano-patterns were fabricated using principles analogous to those indicated by the explanation in Figure 1. The plasmon resonance band of a triangle photomask and FDTD simulation results are shown in Figures 2(e) and 2(f), respectively. When a plasmon resonance at a shorter wavelength than that of the peak wavelength is excited by a laser beam, multipole plasmon resonance is induced. This is similar to what was observed through the FDTD analysis indicated in Figure 1(c). At $0 \mathrm{~s}$ after plasmon excitation (coupled with incident light field) as indicated in Figure 2(f), the relative strong near-field light was observed at the structural tips between the gold triangle structure-resist film interface along the incident polarization direction, which was estimated from the FDTD simulation. Approximately 0.67 fs after plasmon excitation, the scattering component of the light coupled to the gold triangle structure was observed to propagate through the resist film, maintaining the higher order of the LSPR mode whose shape was a triangle inside the resist film, even $(40 \mathrm{~nm})$ from the gold structure-resist film interface (the middle part of the resist film); however, the near-field components could not be seen in the middle of the resist film. Thus, sharp triangle nano-patterns with a depth of $70 \mathrm{~nm}$ could be formed.

Gold was deposited as 7-nm layer on the developed substrate shown in Figure 1(e). In addition, the resist layer was removed using an acetone solution and ultrasonication for 2 min (lift-off process). As a result, gold nanoblocks formed on the glass substrate (Figure 3(a)). Thus, ordered arrays of gold nanoblocks with a periodicity of $300 \mathrm{~nm}$ may be precisely fabricated. The standard deviation of the gold nanoblock side length was estimated to be $3.8 \mathrm{~nm}$. This value matches that obtained from gold nanoblocks fabricated using the high-resolution EB lithography lift-off process previously reported. ${ }^{10}$ In addition, the fabricated gold nanoblocks show an LSPR band around $1200 \mathrm{~nm}$, as shown in Figure 3(b). The reason for the difference in the peak wavelength of the extinction between in Figure 1(b) and in Figure 3(b) can be considered due to the difference of the gold thickness and the structural size. ${ }^{11}$ Figure 3(c) demonstrates that the LSP lithography system combined with a lift-off process can fabricate closely spaced gold nanoblocks. To verify the fabrication of the nanogap gold structures, the extinction spectra of the nanogap gold blocks with different gap widths were measured under parallel (L-mode) and perpendicular (T-mode) polarization for dimer blocks. Figure 3(d) shows the L-mode spectra for nanogap gold blocks with gap widths of $4 \mathrm{~nm}, 18 \mathrm{~nm}, 33$ 

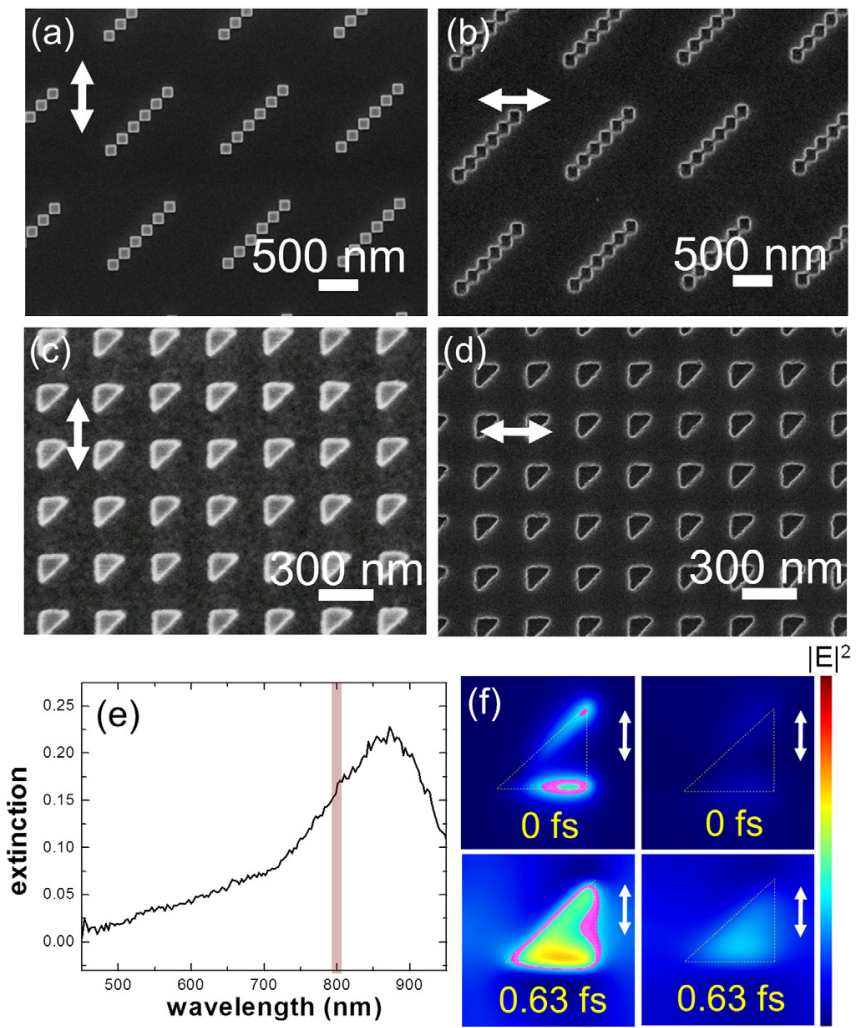

FIG. 2. (Color) SEM images of photomasks and nano-patterns fabricated on a positive photoresist film: (a) a photomask of chains; (b) nano-patterns of chains; (c) a photomask of triangles; (d) nano-patterns of triangles. The inset arrow shows the incident polarization direction during exposure. (e) Extinction spectrum of a triangle photomask. (f) Electromagnetic field distribution between the $\mathrm{Au}$ triangle structure-resist film interface (left side of the figures) and the profile inside the resist film $40 \mathrm{~nm}$ from the Au structure-resist film interface (right side of the figures), $0 \mathrm{fs}$ and $0.67 \mathrm{fs}$ after plasmon excitation, respectively.

$\mathrm{nm}$, and $45 \mathrm{~nm}$. Figure 3(e) shows the gap-width dependence of the peak wavelength in the L- and T-modes. From these figures, LSPR band in the L-mode appears to be red-shifted with a decrease in gap width because dipole-dipole interactions between the two blocks were induced. The T-mode band does not change because EM interaction was not induced. Thus, the lithography system can be used to fabricate nanogap gold structures.

We demonstrated a plasmon-assisted photolithography system based on an exposure principle that can be used to fabricate various sizes and shapes of nano-patterns reflected onto a photomask design. In addition, it is possible to fabricate precise nano-patterns on a positive photoresist that is suitable for a lift-off process. A high-resolution UV nanoimprint technique, however, requires a negative photoresist and, thus, is not appropriate for a lift-off process. The lithography system does not induce any deformation of the nanopattern during mask release. This advantage will become a promising useful tool to replace common nanoimprint technology to produce nano-patterns.
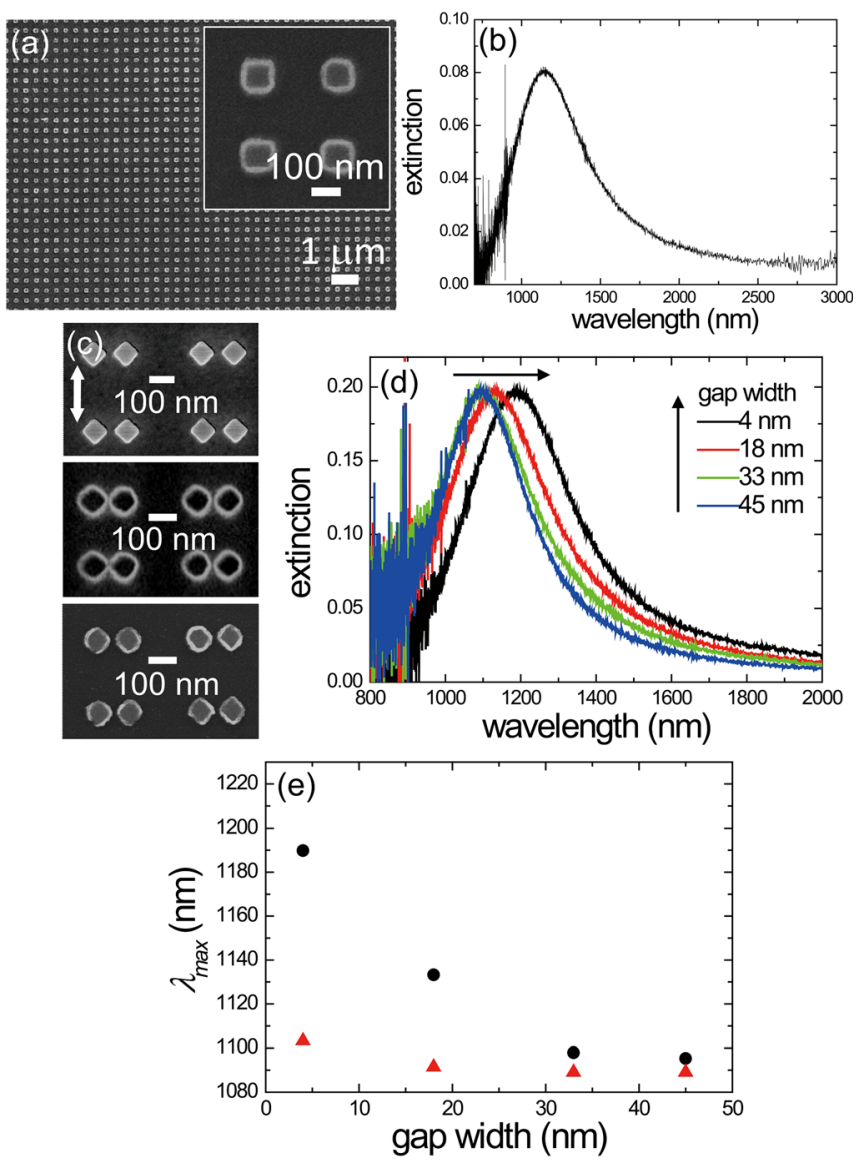

FIG. 3. (Color online) (a) SEM image of gold nanoblocks. The inset shows an enlarged view. (b) Extinction spectrum of the gold nanoblocks. (c) SEM images of photomask (upper side) nano-patterns on the photoresist (center) and gold nanostructures with an 18-nm gap width (bottom). The inset arrow shows the incident polarization direction during exposure. (d) L-mode spectra of nanogap gold blocks with different gap width: (e) Gapwidth dependence on plasmon peak wavelength in L-mode (circle) and T-mode (triangle).

This work was partially supported by funding from the Ministry of Education, Culture, Sports, Science, and Technology of Japan: KAKENHI Grant-in-Aid for Scientific Research on the Priority Area "Strong Photon-Molecule Coupling Fields" (No. 470 (No. 19049001)), No. 23686026 and Grants-in-Aid from Hokkaido Innovation through Nanotechnology Support (HINTS).

${ }^{1}$ O. J. F. Martin, Microelectron. Eng. 67-68, 24 (2003).

${ }^{2}$ W. Srituravanich et al., Nano Lett. 4, 1085 (2004).

${ }^{3}$ D. B. Shao and S. C. Chen, Appl. Phys. Lett. 86, 253107 (2005).

${ }^{4}$ L. Wang et al., Nano Lett. 6, 361 (2006).

${ }^{5}$ T. Kawazoe, T. Takahashi, and M. Ohtsu, Appl. Phys. B 98, 5 (2009).

${ }^{6}$ K. Ueno et al., J. Phys. Chem. Lett. 1, 657 (2010).

${ }^{7}$ K. Ueno et al., J. Am. Chem. Soc. 130, 6928 (2008).

${ }^{8}$ K. Imura et al., J. Am. Chem. Soc. 126, 12730 (2004).

${ }^{9}$ See supplementary material at http://dx.doi.org/10.1063/1.3606505 for the description of the detailed experimental conditions.

${ }^{10}$ K. Ueno et al., J. Am. Chem. Soc. 128, 14226 (2006).

${ }^{11}$ Y. Yokota et al., J. Nanophotonics 1, 011594 (2007). 